\title{
Formation Spectra Of Charmed Meson-nucleus Systems Via $\bar{p}$ Reaction
}

\author{
Junko Yamagata-Sekihara*t \\ National Institute of Technology, Oshima College, Oshima, Yamaguchi, 742-2193, Japan \\ E-mail: yamagata@oshima-k.ac.jp
}

\section{Carmen Garcia-Recio}

Departamento de Física Atómica, Molecular y Nuclear, and Instituto Carlos I de Física Teórica y Computacional, Universidad de Granada, E-18071 Granada, Spain

\section{Juan Nieves}

Instituto de Física Corpuscular (centro mixto CSIC-UV), Institutos de Investigación de Paterna, Aptdo. 22085, 46071, Valencia, Spain

\section{Lorenzo Luis Salcedo}

Departamento de Física Atómica, Molecular y Nuclear, and Instituto Carlos I de Física Teórica y Computacional, Universidad de Granada, E-18071 Granada, Spain

\section{Laura Tolos}

Instituto de Ciencias del Espacio (IEEC/CSIC), Campus UAB, Carrer de Can Magrans s/n, 08193 Cerdanyola del Valles, Spain

Frankfurt Institute for Advanced Studies, Johann Wolfgang Goethe University, Ruth-Moufang-Str. 1, 60438 Frankfurt am Main, Germany

\begin{abstract}
We studied the structure and formation spectra of charmed meson-nucleus systems, with the aim of understanding the charmed meson-nucleon interactions and the properties of the charmed mesons in the nuclear medium. The $\bar{D}$ mesic nuclei are of special interest, since they have tiny decay widths due to the absence of strong decays for the $\bar{D} N$ pair. Employing an effective model for the $\bar{D} N$ and $D N$ interactions and solving the Klein-Gordon equation for $\bar{D}$ and $D$ in finite nuclei, we found that the $D^{-}{ }_{-}^{11} \mathrm{~B}$ system has $1 s$ and $2 p$ mesic nuclear states and that the $D^{0}{ }_{-}^{11} \mathrm{~B}$ system binds in a $1 s$ state. We also calculated the formation spectra of the $\left[D^{-}{ }^{11} \mathrm{~B}\right]$ and $\left[D^{0}{ }_{-}^{11} \mathrm{~B}\right]$ mesic nuclei for an antiproton beam on a ${ }^{12} \mathrm{C}$ target. Our results suggest that it is possible to observe the $2 p D^{-}$mesic nuclear state with an appropriate experimental setup, although its contribution is very small compared to the quasifree contribution.
\end{abstract}

The 26th International Nuclear Physics Conference

11-16 September, 2016

Adelaide, Australia

* Speaker.

${ }^{\dagger}$ A footnote may follow. 


\section{Introduction}

The study of hadron-nucleus systems is one of the ways to provide us with information on the properties of hadron in nuclear medium. The hadron-nucleus bound systems are classified into two categories; one is Coulomb-assisted atomic states and the other is nuclear states generated by the strong interaction. Among them, pionic and kaonic atoms have been intensively investigated over the years[1, 2, 3, 4, 5, 6]. Recently, the properties of charmed mesons in nucleus are very interesting subjects because of improvements of experimental facilities which generates plenty of charmed mesons. One of the first works on charmed mesic nuclei was done in Ref. [7], where the $1 s, 2 s$ and $1 p$ nuclear states of $D^{-}$in ${ }^{208} \mathrm{~Pb}$ were evaluated using the quark-meson coupling model of Ref. [8]. The nuclear energy levels of the $\bar{D}$ meson in ${ }^{208} \mathrm{~Pb}$ and ${ }^{40} \mathrm{Ca}$ were obtained in [9] within a model for the charmed meson-nucleon interaction based on the pion exchange. In addition, $\bar{D} N N$ and $\bar{D}^{*} N N$ bound states were predicted in $[10,11]$ as well as a bound state of $D N N$ in [12].

In this paper, we investigate of the possibility of observing $D^{-}{ }_{-1}^{11} \mathrm{~B}$ and $D^{0}{ }_{-}^{11} \mathrm{~B}$ bound states in ${ }^{12} \mathrm{C}\left(\bar{p}, D^{+}\right)$and ${ }^{12} \mathrm{C}\left(\bar{p}, \bar{D}^{0}\right)$ nuclear reactions. Although we expect that the charmed meson could be bound in nucleus. However, it isn't equal that we could observe the bound states in the formation spectra. The aim of this paper is to show the formation spectra via $\bar{p}$ beam with the Green's function method. This work is based on our recent results [13].

\section{Formulation}

From the charmed meson-nucleon scattering amplitudes and corresponding charmed meson self-energies in Refs. [14, 15, 16], we obtain the optical potential of a meson in the nucleus, which is essential to calculate the formation spectra of the meson-nucleus bound states. Relying on the local density approximation, we evaluate the optical potential for the $D^{-}\left(D^{0}\right)$ mesons. Figs. 1 and 2 show the optical potential with ${ }^{11} \mathrm{~B}$ for $D^{-}$meson and $D^{0}$ meson, respectively. We note that, according to the charmed meson-nucleon scattering amplitudes and self-energies, the present optical potentials we used depend on energy.

We solve the Klein-Gordon equation with the potentials to obtain the bound states.

$$
\left[-\nabla^{2}+\mu^{2}+2 \mu V_{\mathrm{opt}}(r, E)\right] \phi(r)=\left(E-V_{\text {coul }}(r)\right)^{2} \phi(r)
$$

Here, $\mu$ is the meson-nucleus reduced mass and $V_{\text {coul }}(r)$ is the Coulomb potential with a finite nuclear size. For the $D^{0}$ meson case, the Coulomb potential term should be removed. We show the calculated binding energies of $D^{-}$meson and $D^{0}$ meson nuclear bound states for ${ }^{11} \mathrm{~B}$ in Table 1. The atomic states of $D^{-}$meson in ${ }^{11} \mathrm{~B}$ were shown in Table 1 of Ref. [13].

\begin{tabular}{c|cc|cc}
\hline & \multicolumn{2}{|c|}{$D^{-}$meson } & \multicolumn{2}{c}{$D^{0}$ meson } \\
State & binding energy $[\mathrm{MeV}]$ & width $[\mathrm{MeV}]$ & binding energy $[\mathrm{MeV}]$ & width $[\mathrm{MeV}]$ \\
\hline $1 s$ & 21.7 & 0.5 & 6.5 & 10.8 \\
$2 p$ & 14.5 & 2.4 & - & - \\
\hline
\end{tabular}

Table 1: Binding energies and widths of $D^{-}$meson and $D^{0}$ meson-nuclear states in ${ }^{11} \mathrm{~B}$. 


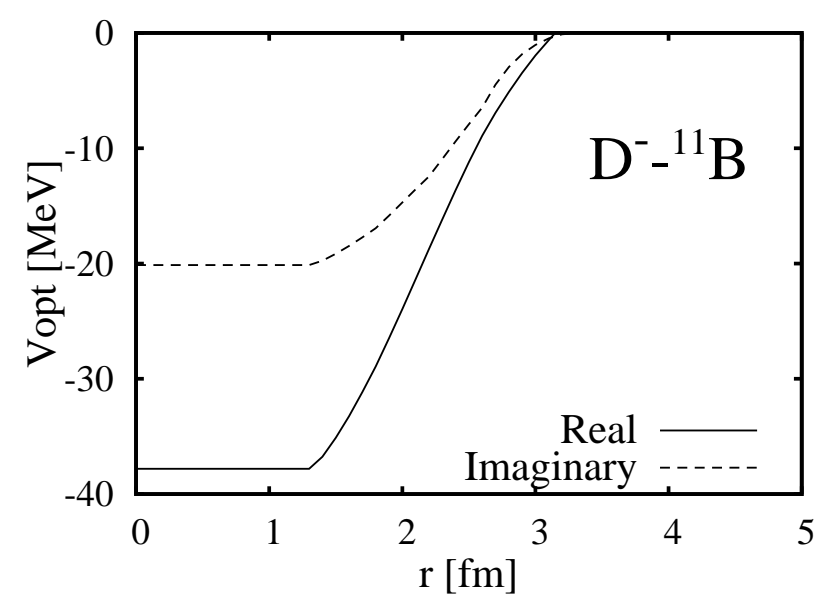

Figure 1: Optical potential for $D^{-}{ }_{-11} \mathrm{~B}$ systems as function of radial $r$ at $E=0[\mathrm{MeV}]$. Solid line is the real part and dashed line is the imaginary part.

These states are produced by the ${ }^{12} \mathrm{C}\left(\bar{p}, D^{+}\right)$and ${ }^{12} \mathrm{C}\left(\bar{p}, \bar{D}^{0}\right)$ reactions. Their formation spectra in this work are calculated in the following manner. First, we separate the cross section into the nuclear response function $S(E)$ and the elementary cross section for the $p(\bar{p}, \bar{D}) D$ reaction within the impulse approximation for $D$ meson production

$$
\left(\frac{d^{2} \sigma}{d \Omega d E_{D}}\right)_{A(\bar{p}, \bar{D})(A-1) \otimes D}=\left(\frac{d \sigma}{d \Omega}\right)_{p(\bar{p}, \bar{D}) D}^{\mathrm{LAB}} \times S\left(E_{D}\right) .
$$

The differential cross section of the elementary process $p(\bar{p}, \bar{D}) D$ in the laboratory frame (LAB), $(d \sigma / d \Omega)_{p(\bar{p}, \bar{D}) D}^{\mathrm{LAB}}$, can be evaluated using some appropriate models or be taken from experimental

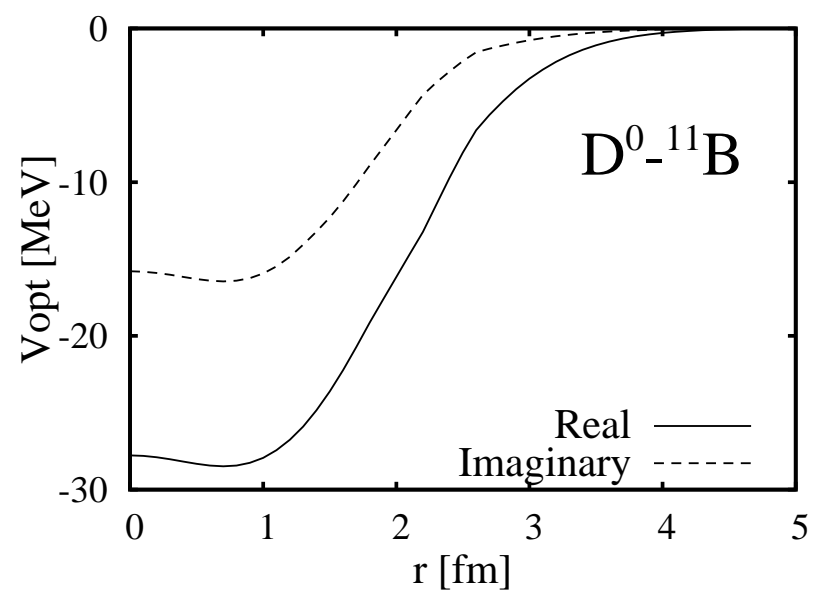

Figure 2: Optical potential for $D^{0}{ }^{11} \mathrm{~B}$ systems as function of radial $r$ at $E=0[\mathrm{MeV}]$. Solid line is the real part and dashed line is the imaginary part. 


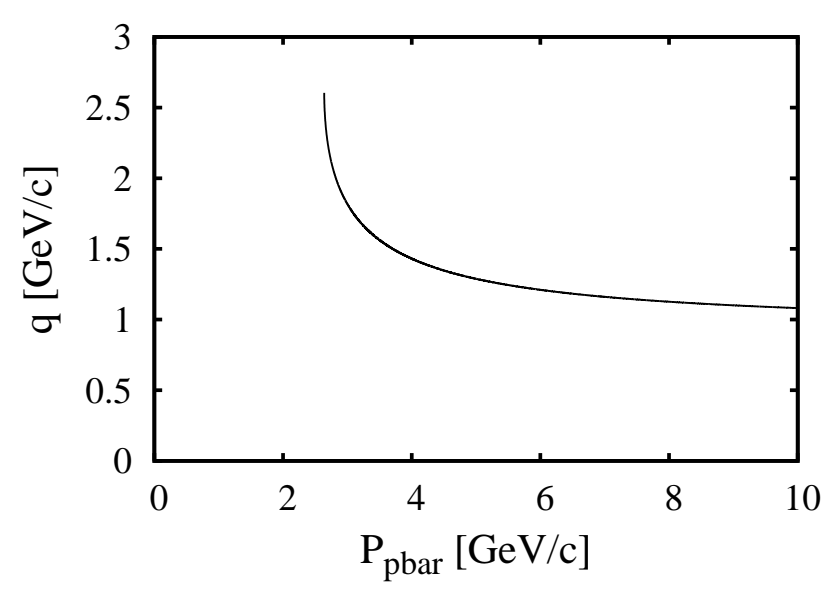

Figure 3: Momentum transfer as a function of the antiproton momentum in the LAB frame.

data. For this cross section, we use the theoretical results of Ref. [17]. The nuclear response function $S\left(E_{D}\right)$ contains information on the dynamics between $D$-meson and the final $(A-1)$ nucleus. To calculate the nuclear response function, we employ the Green's function method. Namely, the nuclear response function with a complex potential is formulated in Ref. [18] as

$$
S\left(E_{D}\right)=-\frac{1}{\pi} \operatorname{Im} \sum_{f} \int d^{3} r d^{3} r^{\prime} \tau_{f}^{\dagger}(\boldsymbol{r}) G\left(E_{D} ; \boldsymbol{r}, \boldsymbol{r}^{\prime}\right) \tau_{f}\left(\boldsymbol{r}^{\prime}\right)
$$

where $\tau_{f}$ denotes the transition amplitude of the initial state $\bar{p}+{ }^{A} Z$ to the proton-hole final nucleus and the outgoing $D-\bar{D}$ meson pair, and $G\left(E_{D} ; \boldsymbol{r}, \boldsymbol{r}^{\prime}\right)$ is the Green's function of the $D$ meson interacting with the nucleus. The summation is taken over all possible final states $f$. The detail of this formulation is written in Ref. [13].

\section{Numerical Results}

We show our numerical results for the formation spectra of the $D^{-}$- and $D^{0}{ }_{-}^{11} \mathrm{~B}$ systems, which are produced in the ${ }^{12} \mathrm{C}\left(\bar{p}, D^{+}\right)$and ${ }^{12} \mathrm{C}\left(\bar{p}, \bar{D}^{0}\right)$ reactions, respectively. We consider forward scattering for the outgoing $D^{+}$or $\bar{D}^{0}$ meson to maximally suppress the momentum transferred to the mesic nuclear bound states. In Fig. 3, we show the momentum transfer in these reactions as a function of the antiproton momentum in the LAB frame. We see the large momentum transfer about $1 \mathrm{GeV} / \mathrm{c}$.

In this work, we use the incident antiproton beam as $8 \mathrm{GeV} / \mathrm{c}$, at which we expect to obtain, both a large elementary cross section [19] and a momentum transfer close to the smallest possible, as seen in Fig. 3. From the theoretical results of Ref. [17], we take $760 \mathrm{nb} / \mathrm{sr}$ for the $p \bar{p} \rightarrow D^{+} D^{-}$ reaction and $40 \mathrm{nb} / \mathrm{sr}$ for the $p \bar{p} \rightarrow D^{0} \bar{D}^{0}$ reaction for the differential cross section of the elementary process $(d \sigma / d \Omega)^{\mathrm{LAB}}$ at forward angle of the emitted $D^{+} / \bar{D}^{0}$ meson. Thus, at $P_{\bar{p}}=8 \mathrm{GeV} / \mathrm{c}$.

Fig. 4 shows the calculated spectrum of ${ }^{12} \mathrm{C}\left(\bar{p}, D^{+}\right)$reaction as a function of the emitted $D^{+}$ meson energy. This reaction produces the $D^{-}$meson bound states. We can see a bump structure 


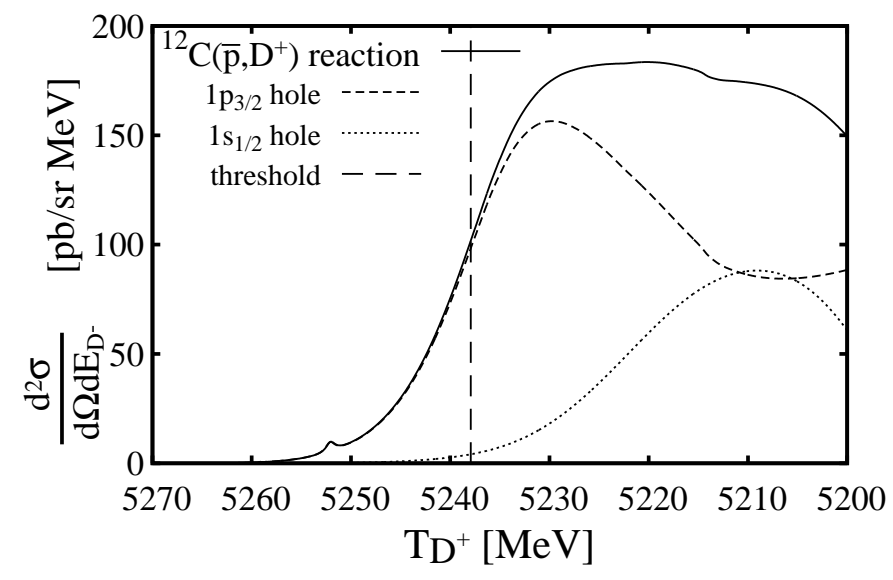

Figure 4: Calculated spectra of ${ }^{12} \mathrm{C}\left(\bar{p}, D^{+}\right)$reaction as a function of the emitted $D^{+}$meson energy at forward angle [13]. The incident antiproton beam is $8 \mathrm{GeV} / \mathrm{c}$. The partial contributions of some shell configurations of the final nucleus are also shown in the figure. The vertical dashed line indicates the $D^{-}$meson production threshold.

around $T_{D^{+}}=5250 \mathrm{MeV}$ placed below the $D^{-}$production threshold. We have checked that this comes from the contribution of the $2 p$ bound state coupled to the $1 p_{3 / 2}$ hole state. However, its contribution is very small compared to the quasifree contribution above the $D^{-}$production threshold, which is caused by the large momentum transfer in the reaction.

Fig. 5 shows the calculated spectrum of ${ }^{12} \mathrm{C}\left(\bar{p}, \bar{D}^{0}\right)$ reaction as a function of the emitted $\bar{D}^{0}$ meson energy. In this spectrum, we do not see any peak structure of the $1 s$ nuclear state listed in Table 1. This is again because of the large momentum transfer of the reaction.

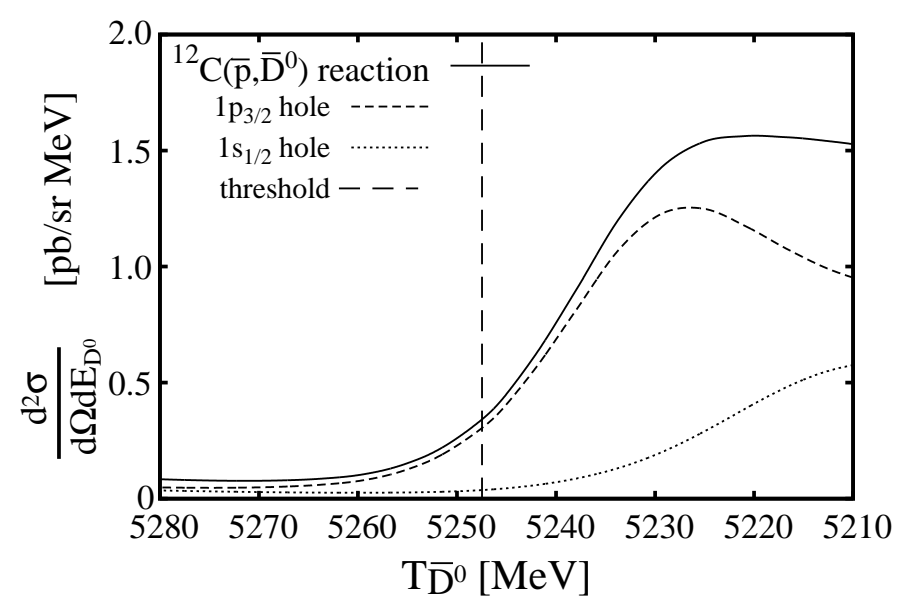

Figure 5: Same as in Fig. 4, but for the ${ }^{12} \mathrm{C}\left(\bar{p}, \bar{D}^{0}\right)$ reaction as a function of the emitted $\bar{D}^{0}$ meson energy at forward angle [13]. 


\section{Summary}

In this work we have calculated the formation spectra of the charmed/anti-charmed mesic nuclear states in the ${ }^{12} \mathrm{C}\left(\bar{p}, D^{+}\right)$and ${ }^{12} \mathrm{C}\left(\bar{p}, \bar{D}^{0}\right)$ antiproton reactions.

First, we have solved the Klein-Gordon equation to obtain the bound states. We have found $1 \mathrm{~s}$ and $2 p$ nuclear states for the $D^{-}$meson and only the $1 s$ level for the $D^{0}$ meson.

Next, we have caluclated the charmed and anti-charmed mesic nuclear formation spectra by employing the Green's function method. The momentum of the antiproton beam has been fixed $8 \mathrm{GeV} / \mathrm{c}$, and the final meson $D^{+} / \bar{D}^{0}$ are taken in the forward direction. We have found that we could see the $2 p D^{-}$bound state in the formation spectrum as a small peak. However, its peak structure is very small compared to the quasifree contribution. For the $D^{0}$ meson case, we couldn't see any peak structures from the bound states. The main reason is the large momentum transfer of this formation reaction.

To see the bound states more clearly, we should consider different production reactions with small momentum transfer. One possibility is to examine the $(\bar{p}, D+N)$ and $(\bar{p}, D+2 N)$ reactions, with a much smaller or even zero momentum transfer, although the formation cross sections could be suppressed as well because of the complexity of the reaction mechanisms.

\section{References}

[1] M. Ericson, T. E. O. Ericson, Annals Phys. 36 (1966) 323.

[2] E. Friedman, A. Gal, Phys. Rept. 452 (2007) 89.

[3] J. Nieves, E. Oset and C. Garcia-Recio, Nucl. Phys. A 554 (1993) 509.

[4] C. Garcia-Recio, J. Nieves, E. Oset, Nucl. Phys. A 547 (1992) 473.

[5] S. Hirenzaki, Mod. Phys. Lett. A 23 (2008) 2497.

[6] H. Gilg, A. Gillitzer, M. Knulle, M. Munch, W. Schott, P. Kienle, K. Itahashi, K. Oyama et al., Phys. Rev. C 62 (2000) 025201.

[7] K. Tsushima, D. H. Lu, A. W. Thomas, K. Saito and R. H. Landau, Phys. Rev. C 59 (1999) 2824.

[8] P. A. M. Guichon, Phys. Lett. B 200 (1988) 235.

[9] S. Yasui and K. Sudoh, Phys. Rev. C 87 (2013) 015202.

[10] S. Yasui and K. Sudoh, Phys. Rev. D 80 (2009) 034008.

[11] Y. Yamaguchi, S. Yasui and A. Hosaka, Nucl. Phys. A 927 (2014) 110.

[12] M. Bayar, C. W. Xiao, T. Hyodo, A. Dote, M. Oka and E. Oset, Phys. Rev. C 86 (2012) 044004.

[13] J. Yamagata-Sekihara, C. Garcia-Recio, J. Nieves, L. L. Salcedo and L. Tolos, Phys. Lett. B 754 (2016) 26.

[14] L. Tolos, C. Garcia-Recio and J. Nieves, Phys. Rev. C 80 (2009) 065202.

[15] C. Garcia-Recio, J. Nieves and L. Tolos, Phys. Lett. B 690 (2010) 369.

[16] C. Garcia-Recio, J. Nieves, L. L. Salcedo and L. Tolos, Phys. Rev. C 85 (2012) 025203.

[17] J. Haidenbauer and G. Krein, Phys. Rev. D 89 (2014) 114003.

[18] O. Morimatsu and K. Yazaki, Nucl. Phys. A 435 (1985) 727.

[19] A. B. Kaidalov and P. E. Volkovitsky, Z. Phys. C 63 (1994) 517. 